\title{
The Absence of Fever or Leukocytosis Does Not Exclude Infection Following Cranioplasty
}

\author{
Fady Girgis, Brian P. Walcott, Churl-Su Kwon, Sameer A. Sheth, Wael Asaad, \\ Brian V. Nahed, Emad N. Eskandar and Jean-Valery Coumans
}

\begin{abstract}
Background: Cranioplasty encompasses various cranial reconstruction techniques that are used following craniectomy due to stroke or trauma. Despite classical infectious signs, symptoms, and radiologic findings, however, the diagnosis of infection following cranioplasty can be elusive, with the potential to result in definitive treatment delay. We sought to determine if fever or leukocytosis at presentation were indicative of infection, as well as to identify any factors that may limit its applicability. Methods: Following institutional review board approval, a retrospective cohort of 239 patients who underwent cranioplasty following craniectomy for stroke or trauma was established from 2001-2011 at a single center (Massachusetts General Hospital). Analysis was then focused on those who developed a surgical site infection, as defined by either frank intra-operative purulence or positive intra-operative cultures, and subsequently underwent operative management. Results: In 27 total cases of surgical site infection, only two had a fever and four had leukocytosis at presentation. This yielded a false-negative rate for fever of $92.6 \%$ and for leukocytosis of $85.2 \%$. In regard to infectious etiology, $22(81.5 \%)$ cases generated positive intra-operative cultures, with Propionibacterium acnes being the most common organism isolated. Median interval to infection was 99 days from initial cranioplasty to time of infectious presentation, and average follow-up was 3.4 years. Conclusions: The utilization of fever and elevated white blood cell count in the diagnosis of post-cranioplasty infection is associated with a high false-negative rate, making the absence of these features insufficient to exclude the diagnosis of infection.
\end{abstract}

RÉSUMÉ: L'absence de fièvre ou d'hyperleucocytose n'exclut pas la présence d'infection après une cranioplastie. Contexte: Plusieurs techniques de cranioplastie peuvent être utilisées pour la reconstruction crânienne après une craniectomie effectuée suite à un accident vasculaire cérébral ou à un traumatisme. Cependant, malgré les signes classiques d'infection, les symptômes et les signes radiologiques, le diagnostic d'infection après une cranioplastie peut échapper à la détection, ce qui peut en retarder le traitement. Nous avons cherché à déterminer si la fièvre ou l'hyperleucocytose au moment de la consultation étaient des indicateurs de l'infection et à identifier tout facteur qui pourrait limiter son applicabilité. Méthode: Le protocole a été approuvé par le comité d'éthique de la recherche de l'institution. L'étude a porté sur une cohorte rétrospective de 239 patients traités entre 2001 et 2011 , qui avaient subi une cranioplastie après une craniectomie effectuée pour traiter un AVC ou un traumatisme dans un même centre, le Massachusetts General Hospital. L'analyse a ciblé les patients qui avaient présenté une infection de la zone opératoire, définie comme étant la présence de pus ou de cultures positives au niveau de la zone opératoire, et qui ont subi un traitement chirurgical. Résultats: Parmi les 27 cas d'infection au niveau de la zone opératoire, seulement 2 patients présentaient de la fièvre et 4 avaient une hyperleucocytose au moment de la consultation. Le taux de faux-négatif pour la fièvre était donc de $92,6 \%$ et pour l'hyperleucocytose de $85,2 \%$. Quant à l'étiologie de l'infection, les cultures de la zone opératoire étaient positives chez 22 patients (81,5\%) et le Propionibacterium acnes était l'agent infectieux le plus souvent impliqué. L'intervalle médian entre la cranioplastie initiale et la consultation pour l'infection était de 99 jours et le suivi moyen de 3,4 ans. Conclusions: L'utilisation de la fièvre et d'un décompte élevé de leucocytes pour diagnostiquer l'infection après une cranioplastie est associée à un taux élevé de faux-négatifs, ce qui rend l'absence de ces éléments insuffisante pour exclure le diagnostic d'infection.

Keywords: cranioplasty, trauma, infections of the nervous system, complication, craniofacial surgery

doi:10.1017/cjn.2015.46

Can J Neurol Sci. 2015; 42: 255-259

Infection is a well-documented complication of cranioplasty, occurring in $3.7-16.7 \%$ of cases. ${ }^{1-8}$ As delayed recognition can potentially result in significant morbidity and mortality, early detection of these post-operative episodes is crucial. Leakage from the incision site, swelling of tissue overlying the flap, and exposed hardware are all common presenting signs suggesting the

From the Department of Neurosurgery (FG), University of Calgary, Foothills Hospital, Calgary AB, Canada; Department of Neurosurgery (BPW, CK, SAS, BVN, ENE, JC),

Massachusetts General Hospital and Harvard Medical School, Boston, Massachusetts; Department of Neurosurgery and the Brown Institute for Brain Science (WA), Brown

University Alpert Medical School and Rhode Island Hospital, Providence, Rhode Island, USA.

Received July 26, 2014. Final Revisions Submitted January 30, 2015.

Correspondence to: Fady Girgis, Department of Neurosurgery, University of Calgary, Foothills Medical Centre, $12^{\text {th }}$ Floor, 1403,29 St NW, Calgary, AB T2N 2T9.

Email: fadygirgis@yahoo.com 
presence of localized infection following this procedure. Systemic signs, however, are not as readily distinguishable, making their use in the diagnosis and management of this complication uncertain.

Systemic evidence of the early stages of infection can be summarized in general terms using the features of the Systemic Inflammatory Response Syndrome (SIRS), where diagnosis requires the presence of two of four signs: fever, leukocytosis, tachypnea, and tachycardia. ${ }^{9}$ Studies looking specifically at the role of elements from this syndrome in central nervous system infections are scarce but existing evidence does lean towards a greater utility in acute, more so than chronic, infectious processes $^{10,11}$ The utility of SIRS elements in post-cranioplasty infection, however, has not been directly addressed in the literature. Therefore, in this study, we focused on two commonly recorded signs in patients presenting with suspected infection after cranioplasty, fever and leukocytosis, in order to determine their ability to predict subsequently confirmed infection. In addition, we sought to identify any factors that may limit their applicability.

\section{Methods}

After obtaining Institutional Review Board approval, a retrospective review was conducted to identify all patients who had undergone cranioplasty at the Massachusetts General Hospital from 2001 to 2011. Of the 239 cases identified, 27 underwent further surgery for suspicion of post-operative infection. Infection was defined either by evidence of frank purulence as described in operative records and/or the presence of positive intra-operative cultures from a non-superficial source, such as subcutaneous tissue, bone, or epidural, subdural, and/or intracranial collections.

In addition to characterizing factors such as age and gender, the following data was collected from patient records: The indication for and type of craniectomy performed; the method of bone flap preservation, if applicable; the use of hardware during treatment such as a shunt or extra-ventricular drain; and the type of bone flap implanted during cranioplasty. In regard to infectious documentation, the following data was collected on the date of infectious presentation after cranioplasty: The white blood cell (WBC) count and forehead temperature as measured using a temporal thermometer; the presence of exposed hardware or bone; and the presence of wound leakage. Further information collected included blood culture and non-superficial intra-operative culture results. Although pain at the site is classically described with postoperative infections, pain scores were not recorded or analyzed, as the data was considered to be too subjective.

The type of craniectomy was classified as convexity or bifrontal, while the indication for craniectomy was classified as either trauma or stroke. Bone flap preservation, when applicable, was classified as in vivo or frozen, while the type of bone flap implanted was classified as either autologous or synthetic. Infectious presentation was defined as the date the patient presented to hospital with infectious symptoms. On the same date, blood was drawn for WBC count and culture, temperature was measured to assess for fever and the incision was inspected for exposed hardware or bone and leakage. An elevated WBC count was defined as $\geq 11.0 \times 10^{9} / \mathrm{L}$ and fever was defined as a temporal temperature $\geq 101.3^{\circ} \mathrm{F}\left(\geq 38.5^{\circ} \mathrm{C}\right)$, which are the cutoffs used at this center.

Statistical analysis was performed using the $\mathrm{R}$ programming environment (Vienna, Austria). A $p$ value of less than 0.05 was pre-defined to indicate a statistically significant result.

\section{Table 1: Clinical parameters}

\begin{tabular}{|c|c|}
\hline Median age, years (range) & $51.0(0.4-67.5)$ \\
\hline \multicolumn{2}{|l|}{ Indication for craniectomy } \\
\hline Trauma & $12 / 27(44.4 \%)$ \\
\hline Stroke & $15 / 27(55.6 \%)$ \\
\hline \multicolumn{2}{|l|}{ Type of craniectomy performed } \\
\hline Convexity & $25 / 27(92.6 \%)$ \\
\hline Bifrontal & $2 / 27(7.4 \%)$ \\
\hline \multicolumn{2}{|l|}{ Bone flap preservation } \\
\hline Subcutaneous & $7 / 27(25.9 \%)$ \\
\hline Freezing & $15 / 27(55.6 \%)$ \\
\hline Not preserved & $5 / 27(18.5 \%)$ \\
\hline $\begin{array}{l}\text { Median interval from cranioplasty to infectious presentation, } \\
\text { days (range) }\end{array}$ & $99.0(1-2919)$ \\
\hline
\end{tabular}

\section{ReSUlTS}

A total of 239 cranioplasty patients were identified, with $27(11.3 \%)$ of these undergoing repeat surgeries for the treatment of infection. Infection was suspected based on a combination of clinical and radiographic factors, including swelling around the incision site, wound drainage or breakdown, leukocytosis, and/or fluid collections on computed tomography or magnetic resonance images (MRI). Infection was diagnosed in all suspected cases and was defined either by positive intra-operative cultures and/or frank purulence reported in the operative records. In all cases, the bone flap was removed and discarded and intravenous antibiotics were given for a minimum of six weeks treatment duration.

Of the patients that developed infection, the median age at time of craniectomy was 51.0 (range $0.4-67.5$ ), and $63.0 \%(17 / 27$ ) of patients were male. Indications for craniectomy included trauma in $44.4 \%(12 / 27)$ of patients, and stroke for the remainder $(15 / 27$; $55.6 \%)$. Of the craniectomies performed, $92.6 \%$ (25/27) consisted of unilateral convexity bone removal and the remaining $7.4 \%$ $(2 / 27)$ were bifrontal craniectomies. The bone flap was preserved in 22 of the 27 cases either in vivo by abdominal subcutaneous implantation $(7 / 27,25.9 \%)$, or by freezing $(15 / 27,55.6 \%)$. In the $18.5 \%(5 / 27)$ of cases where it was not preserved, a synthetic bone flap was inserted for reconstruction of the skull defect. The median interval from the date of cranioplasty to the date of presentation with infectious symptoms was 99 days, with values ranging from 1 to 2919 days. A summary of clinical parameters can be found in Table 1.

At presentation to hospital for infectious symptoms, $14.8 \%$ of patients $(4 / 27)$ had a WBC count $\geq 11.0 \times 10^{9} / \mathrm{L}$, and $7.4 \%(2 / 27)$ had a temporal temperature $\geq 101.3^{\circ} \mathrm{F}$. In terms of the appearance of the incision, $18.5 \%$ of patients $(5 / 27)$ had exposed hardware or bone visible through the incision, and 51.9\% (14/27) had drainage from the wound. Blood cultures were drawn in 26 of the 27 patients and all returned no growth. Non-superficial intracranial cultures, meaning from subcutaneous tissues or deeper, were collected during surgery in 25 of the 27 patients, with 22 of these $(81.5 \%)$ yielding positive cultures. Seven organisms were isolated in total, with the tissue collected from some patients growing more than one organism. The most frequently encountered organism was Propionibacterium acnes. A summary 
Table 2: Details on infections

\begin{tabular}{|c|c|c|}
\hline Patient characteristics at infectious presentation & & \\
\hline WBC white blood cell count $\geq 11.0 \times 10^{9} / \mathrm{L}$ & $4 / 27(14.8 \%)$ & \\
\hline Temperature $\geq 101.3^{\circ} \mathrm{F}$ & $2 / 27(7.4 \%)$ & \\
\hline Presence of exposed hardware or bone & $5 / 27(18.5 \%)$ & \\
\hline Presence of wound drainage & $14 / 27(51.9 \%)$ & \\
\hline \multicolumn{3}{|c|}{ Organisms isolated from intra-operative cultures, number of occurrences* } \\
\hline & Propionibacterium acnes & $7 / 26(26.9 \%)$ \\
\hline & Methicillin-resistant Staphylococcus aureus & $6 / 26(23.1 \%)$ \\
\hline & Methicillin-sensitive Staphylococcus aureus & $5 / 26(19.2 \%)$ \\
\hline & Coagulase-negative Staphylococcus & $5 / 26(19.2 \%)$ \\
\hline & Citrobacter sp. & $1 / 26(3.8 \%)$ \\
\hline & Proteus sp. & $1 / 26(3.8 \%)$ \\
\hline & Escherichia coli & $1 / 26(3.8 \%)$ \\
\hline
\end{tabular}

* Some operations yielded more than one organism

of the infectious characteristics of the studied patients can be found in Table 2.

Of the 27 patients with infection, 8 had intra-axial collections, 18 had subdural collections, and 1 patient had an epidural collection. Of the patients who had either leukocytosis (four patients) or fever (two patients) at presentation, none had intraaxial collections, five had subdural collections, and one had an epidural collection. Fourteen of 27 patients $(51.9 \%)$ underwent MRI with diffusion weighting, with seven of these (50\%) showing restricted diffusion at the infected site. Overall median follow-up time was 29.7 months (range 1.7-122.7).

In this series, the false-negative rate was $92.6 \%$ for fever and $85.2 \%$ for leukocytosis. Univariate logistic regression analysis revealed no significant predictors of false-negative outcomes for these two variables (Table 3).

\section{Discussion}

Similar to the diagnosis of infection in other post-surgical patients, fever and leukocytosis are commonly available tests utilized by the neurosurgeon in the investigation of post-cranioplasty infection. We have shown, however, that the utility of a high fever or elevated WBC count can be deceiving, in that they carry a

Table 3: Predictors of false-negative leukocytosis on presentation by univariate analysis

\begin{tabular}{l|c}
\hline Factor & P-value \\
\hline Gender & 0.128 \\
\hline Type of implant & 0.204 \\
\hline Presence of hardware & 0.269 \\
\hline Exposed hardware & 0.561 \\
\hline Location & 1.0 \\
\hline Indication for craniectomy & 1.0 \\
\hline Wound drainage & 1.0 \\
\hline Indication for craniectomy & 1.0 \\
\hline
\end{tabular}

false-negative rate of $92.6 \%$ and $85.2 \%$, respectively. Unfortunately, a small sample size prevented the finding of any significant factors that may predict outcomes based on these variables.

This lack of systemic signs such as leukocytosis and fever in the presentation of post-cranioplasty infection mirrors the relative lack of these findings in other central nervous system infections. Vates ${ }^{10}$ reported on 24 cases of intra-operatively diagnosed pituitary abscess, where less than one third of patients presented with systemic signs and symptoms and most presented with findings related to mass effect. Another example is that of Candon, ${ }^{11}$ who reviewed 73 cases of intramedullary spinal cord abscesses. The authors found that patients who presented with less than one week of symptoms, the 'acute-onset group', were more likely to have fever and/or leukocytosis, as compared to the 'chronic group', who presented with greater than six weeks of symptoms. This is similar to our study, in that most patients described an insidious onset of symptoms rather than an acute event, making systemic findings less likely.

Another reason for such a low incidence of fever and leukocytosis in post-cranioplasty infections could be that neurosurgical patients are frequently on medications that may mask infectious signs and symptoms. These include corticosteroids and chemotherapy, the use of which can cause immunosuppression and prevent the body from mounting an appropriate immune response. However, no patients in this series were receiving longterm corticosteroids or chemotherapy. Alternatively, the low rates of systemic response may be related to the pathogenic mechanism involved in the development of the infection. Blunting of the inflammatory response, that is, the recruitment of neutrophils and other plasma components to the surgical site, may occur due to the isolated location of the infection, as purulent fluid collections in these cases are often localized in the form of an abscess. ${ }^{12}$

Other evidence for lack of systemic involvement in cranioplasty infection is that all patients in this study had negative blood cultures, a trend that questions the need for this investigation in this subset of patients. A recent study investigated the general need for blood cultures in patients with suspected bacteremia, concluding that isolated fever or leukocytosis alone are not sufficient to predict bacteremia, while SIRS is a sensitive but 
non-specific predictor ${ }^{13}$ Our results support this conclusion in that all but one patient met criteria for SIRS, while all patients tested negative for bacteremia. Based on these results, it may be likely that blood cultures are not warranted in cases of post-cranioplasty infection, unless the patient is exhibiting systemic signs of disease such as SIRS or sepsis. Furthermore, specific scoring systems for SIRS and its relation to the underlying etiology may help predict outcomes for those patients in critical care settings ${ }^{14,15}$ and post-discharge surveillance systems may provide quality assurance in surgical trauma patients. ${ }^{16}$

The pre-test probability of any diagnostic test is related to the disease prevalence, therefore knowledge factors that influence infection rates must be taken into consideration for each clinical scenario. ${ }^{17}$ There have been multiple attempts to identify predictors of infection development following cranioplasty. For one, the timing of cranioplasty relative to the initial craniectomy has been suggested to influence the subsequent development of infection. For those undergoing "delayed" cranioplasty (studies varying from 3-12 months following craniectomy), infection rates were lower. ${ }^{6,7,18}$ However, one contrasting study demonstrated that waiting a shorter period of time, from zero to six months, resulted in lower infection rates. ${ }^{1}$ A systematic review concluded that there was no statistically significant association between the interval to cranioplasty and subsequent infection. ${ }^{19}$ Additionally, it has been hypothesized that the type of cranioplasty material and/or storage method may influence infection rates, although the results are conflicting. Inamasu reported that in patients whose craniectomy was subsequent to a traumatic injury, autologous bone flaps that were cryopreserved had higher infection rates after cranioplasty than those stored subcutaneously. ${ }^{4}$ Another study, by Matsuno, found that frozen autologous and polymethylmethacrylate (PMMA) implants had higher infection rates compared to titanium mesh implants $\left(38.5 \%\right.$ and $6.3 \%$ vs. $0 .^{5}$ Conversely, Chang found that frozen autologous implants were $13.8 \%$ less likely to get an infection compared to titanium mesh and PMMA. ${ }^{1}$ Similar to the timing of surgery, a systematic review in 2011 by Yadla found no significant difference between autograft and allograft materials ${ }^{19}$ Interestingly, four studies investigating the feasibility of re-implanting an infected autograft after meticulous debridement, scrubbing with iodine solution and followed by intravenous antibiotics, showed success in 69-100\% of cases ${ }^{2,20-22}$ Further study is needed to identify predictors of cranioplasty infection, the results of which may also optimize diagnostics.

Of the patients in our study who underwent an MRI scan, 50\% had restricted diffusion at the infection site. The only study looking at diffusion weighting in the diagnosis of postcranioplasty infection reports it to be a useful investigation, ${ }^{23}$ however our results are clearly conflicting. Despite being useful in the differentiation of abscesses from tumors ${ }^{24}$ restricted diffusion in central nervous system infections is associated with a high false-negative rate, especially when the infection is located extradurally. ${ }^{25}$ Although not performed on any of the patients included in this study, white blood cell tagged scans, such as indium-111 or gallium scans, can be helpful in the diagnosis of cranial infection. Considering these nuclear medicine investigations are classically described in the diagnosis of osteomyelitis, ${ }^{26,27}$ it would be interesting to see if their utility is maintained in infections of bone that is re-inserted into the body, as in the setting of cranioplasty infections.
The main limitations of this study are its small sample size and retrospective nature. To maximize accuracy, the database used to identify patients was confirmed by comparing surgeon records with anesthesia and billing records. Cases of suspected cranioplasty infection not undergoing operative intervention would be useful to analyze, however there was no accurate means of identifying these patients with a retrospective study design. The results do show a convincing trend in the high false-negative rates of fever and leukocytosis in this patient subset. An ongoing, prospectively identified cohort is now established based on these preliminary findings.

\section{Conclusion}

The utilization of fever and elevated WBC count in the diagnosis of post-cranioplasty infection is associated with a high falsenegative rate, making the absence of these features insufficient to exclude the diagnosis of infection. This information may serve useful for practitioners placing a high importance on these factors, as surgical treatment should be tailored based on the clinical suspicion of infection, rather than systemic signs.

\section{Disclosures}

All authors have seen and approved this manuscript and have no disclosures to report. No portion of this work has been previously published or presented. Fady Girgis, Brian Walcott, Churl-Su Kwon, Sameer Sheth, Wael Assad, Brian Nahed, Emad Eskandar, and Jean-Valery Coumans declare they have no conflict of interest.

\section{REFERENCES}

1. Chang V, Hartzfeld P, Langlois M, Mahmood A, Seyfried D. Outcomes of cranial repair after craniectomy. J Neurosurg. 2010; 112:1120-4.

2. Chiang H-Y, Steelman VM, Pottinger JM, et al. Clinical significance of positive cranial bone flap cultures and associated risk of surgical site infection after craniotomies or craniectomies. J Neurosurg. 2011;114:1746-54.

3. Gooch MR, Gin GE, Kenning TJ, German JW. Complications of cranioplasty following decompressive craniectomy: analysis of 62 cases. Neurosurg. 2009;26:E9.

4. Inamasu J, Kuramae T, Nakatsukasa M. Does difference in the storage method of bone flaps after decompressive craniectomy affect the incidence of surgical site infection after cranioplasty? Comparison between subcutaneous pocket and cryopreservation. J Trauma. 2010;68:183-7; discussion 7.

5. Matsuno A, Tanaka H, Iwamuro H, et al. Analyses of the factors influencing bone graft infection after delayed cranioplasty. Acta Neurochir (Wien). 2006;148:535-40; discussion 40

6. Rish BL, Dillon JD, Meirowsky AM, et al. Cranioplasty: a review of 1030 cases of penetrating head injury. Neurosurgery. 1979;4:381-5.

7. Thavarajah D, De Lacy P, Hussien A, Sugar A. The minimum time for cranioplasty insertion from craniectomy is six months to reduce risk of infection-a case series of 82 patients. Br J Neurosurg. 2012;26:78-80.

8. Vahtsevanos K, Triaridis S, Patrikidou A, et al. The Atkinson Morley's Hospital joint neurosurgical-maxillofacial procedures: cranioplasty case series 1985-2003. J Craniomaxillofac Surg. 2007;35:336-42.

9. American College of Chest Physicians/Society of Critical Care Medicine Consensus Conference: definitions for sepsis and organ failure and guidelines for the use of innovative therapies in sepsis. Critical Care Medicine. 1992;20:864-74.

10. Vates GE, Berger MS, Wilson CB. Diagnosis and management of pituitary abscess: a review of twenty-four cases. J Neurosurg. 2001;95:233-41. 
11. Candon E, Frerebea P. Bacterial abscesses of the spinal cord. Review of the literature (73 cases). Revue Neurol Paris. 1994;150:370-6.

12. Jovanovic Z, Illic M, Jankovic S. Pathogenic mechanisms in the development of surgical site infections. Medicinski Pregled. 2007;60:343-50.

13. Coburn B, Morris AM, Tomlinson G, Detsky AS. Does this adult patient with suspected bacteremia require blood cultures? JJAMA. 2012;308:502-11.

14. Han J, Liang HP. Clinical significance of scoring system for systemic inflammatory response syndrome. Chin J of Traumatol. 2006; 9:316-20.

15. Brun-Bruisson $\mathrm{C}$. The epidemiology of the systemic inflammatory response. Intensive Care Med. 2000;26:S64-74.

16. McIntyre LK, Warner KJ, Nester TA, Nathens AB. The incidence of post-discharge surgical site infection in the injured patient. J Trauma. 2009;66:407-10.

17. Walcott BP, Redjal N, Coumans JVCE. Infection following operations on the central nervous system: deconstructing the myth of the sterile field. Neurosurg. 2012;33:8.

18. Cheng Y-K, Weng H-H, Yang J-T, Lee M-H, Wang T-C, Chang C-N. Factors affecting graft infection after cranioplasty. J Clin Neurosci. 2008;15:1115-9.

19. Yadla S, Campbell PG, Chitale R, Maltenfort MG, Jabbour P, Sharan AD. Effect of early surgery, material, and method of flap preservation on cranioplasty infections: a systematic review. Neurosurgery. 2011;68:1124-9; discussion 30 .
20. Auguste KI, McDermott MW. Salvage of infected craniotomy bone flaps with the wash-in, wash-out indwelling antibiotic irrigation system. Technical note and case series of 12 patients. J Neurosurg. 2006;105:640-4.

21. Bruce JN, Bruce SS. Preservation of bone flaps in patients with postcraniotomy infections. J Neurosurg. 2003;98:1203-7.

22. Widdel L, Winston KR. Pus and free bone flaps. J Neurosurg Pediatrics. 2009;4:378-82.

23. Tamaki T, Eguchi T, Sakamoto M, Teramoto A. Use of diffusionweighted magnetic resonance imaging in empyema after cranioplasty. Br J Neurosurg. 2004;18:40-4.

24. Xu XX, Li B, Yang HF, Du Y, Li Y, Wang WX. Can diffusionweighted imaging be used to differentiate brain abscess from other ring-enhancing brain lesions? A meta-analysis. Clin Radiol. 2014;69:909-15.

25. Farrell CJ, Hoh BL, Pisculli ML, Henson JW, Barker II FG, Curry WT. Limitations of diffusion-weighted imaging in the diagnosis of postoperative infections. Neurosurg. 2008;62: 577-583.

26. Bruni C, Padovano F, Travascio L, Schillaci O, Simonetti G. Usefulness of hybrid SPECT/CT for the 99mTc-HMPAO-labeled leukocyte scintigraphy in a case of cranial osteomyelitis. Braz $\mathbf{J}$ Infect Dis. 2008;12:558-60.

27. Liberatore M, Drudi FM, Tarantino R, Prosperi D, Fiore V, Missori P. Tc-99m exametazime-labeled leukocyte scans in the study of infections in skull neurosurgery. Clin Nucl Med. 2003;28:971-4. 